\title{
Analysis of Car Travel Characteristics Based on Vehicle OBD Data
}

\section{Sun Jianping 1, 2, 3, 4, Guo Jifu', 2, 3, 4, Wen Huimin², 3, 4, Zhao Xiang 2, 3, 4, Lei} Fangshu' 2,3

1Beijing Jiaotong University, Beijing 100044, China;

${ }^{2}$ Beijing Transport Institute, Beijing 100073, China

${ }^{3}$ Beijing Key Laboratory of Urban Traffic Operation Simulation and Decision Support 100073, China ${ }^{4}$ Beijing International Science and Technology Cooperation Base of Urban Traffic 100073, China

aemail: guojf@bjtrc.org.cn, bemail: sunjp@bjtrc.org.cn, cemail: wenhm@bjtrc.org.cn, demail: zhaox@bjtrc.org.cn, eemail: leifs@bjtrc.org.cn

Keywords: traffic management; car travel characteristic; OBD data; user portrayal

Abstract: Car travel characteristics is one of the important factors affecting of the urban road network operation. To provide support for traffic refinement management and policy making, collecting car travel data and having in depth analysis of its travel behavior characteristics, is the key aspect in encouraging car travel behavior change and reducing the vehicle use intensity. Based on the travel data and track data collecting by the vehicle OBD data, car travel characteristics analysis method is put forward, and the quantitative indicators such as trip rate, travel frequency, travel distance can be get, and the travel index variation characteristics were analyzed under the different stages, different regions and different policy. Studying the travel rule by using the big data analysis method, on a deep level, traffic characteristics of user portrait, road network non-linear coefficient can be further obtained. The analysis results show that the intelligent driving data can better reflect the characteristics of the car travel behavior, and make quantitative evaluation of the implementation of the transport demand management policies.

\section{Introduction}

In China, the urban traffic congestion has got increasingly severer amid the continuous expansion of vehicle base over the past years. This makes it an important direction to reduce the intensity of vehicle use and increase the green traffic weight under the traffic development strategies of many metropolises. Moreover, it is urgent to identify the rule of mobility of traffic participants and seek a sound solution for the traffic disease. After the outset of the big data era, traffic big data are emerging and making characteristics and problems of traffic increasingly explicit and visible. For example, we can parse the spatial relation between the workplace and the residence of urban residents based on mobile phone signaling data[1] and study mobility characteristics of the floating population[2]. We can collect the public traffic information based on IC card data[3] and forecast the passenger flow of public traffic[4]. Moreover, we can realize real-time monitoring over traffic condition based on floating vehicle data and forecast recurrent congestion areas in advance[5]. Meanwhile, the emerging sharing traffic has generated mass mobility data, which will also help describe trip characteristics more precisely, while changing the service mode of the traditional traffic industry.

We have accumulated a vast amount of traffic data and research results relating to traffic flow, transit, taxi, other commercial vehicles, residents' mobility and other aspects. However, we should still need to improve the collection and analysis of mobility data of cars, which are the biggest 
contributor to the road congestion. Many private cars run in the road network but we lack data on their rule of mobility and characteristics of use as well as the track of macroscopic changes in the urban traffic. As a result, we will be difficult to support the comprehensive policies aimed to reduce the intensity of vehicle use. The studies on mobility characteristics of cars so far are mainly based on the traditional resident's mobility survey and focus on mobility characteristics[6], mobility mode[7], influencing factors[8-10] and other aspects. Yet, these studies are limited to some extent in respect of data size of survey, integrity of information and dynamic timeliness, which makes it impossible to present mobility characteristics of cars in depth and accurately. Therefore, we still don't have effective data and methodologies necessitated to perform a quantitative analysis of the impact on mobility conducts from the demand management policy made by the traffic decision-maker for mobility of cars, oil price change, fare adjustment of public traffic, online taxi service, sharing bicycle and other factors. In this paper, we will study the mobility rule of cars based on trip data collected from the on-board diagnostic (OBD) system (intelligent driving data) and take these data of Beijing in 2016 for example to provide a vigorous support for reasonable guidance of car use and determination of control measures.

\section{Collection and Processing Technologies for Car Trips Data}

\section{Data collection}

OBD data of cars are principally obtained from the "Intelligent Driving Box" installed on the cars. The Intelligent Driving Box based on OBD data is a CAN bus device that can collect the trip data, positioning data, vehicle status, fuel consumption, driving conduct and engine operation parameters of cars (see Figure 1) in a real-time manner. It can remind the driver to improve their driving conducts and realize fuel economy, emission reduction and safety. Moreover, the device can record continuous and detailed data of the car and the driver, and these data are the most important part of the traffic data cloud.

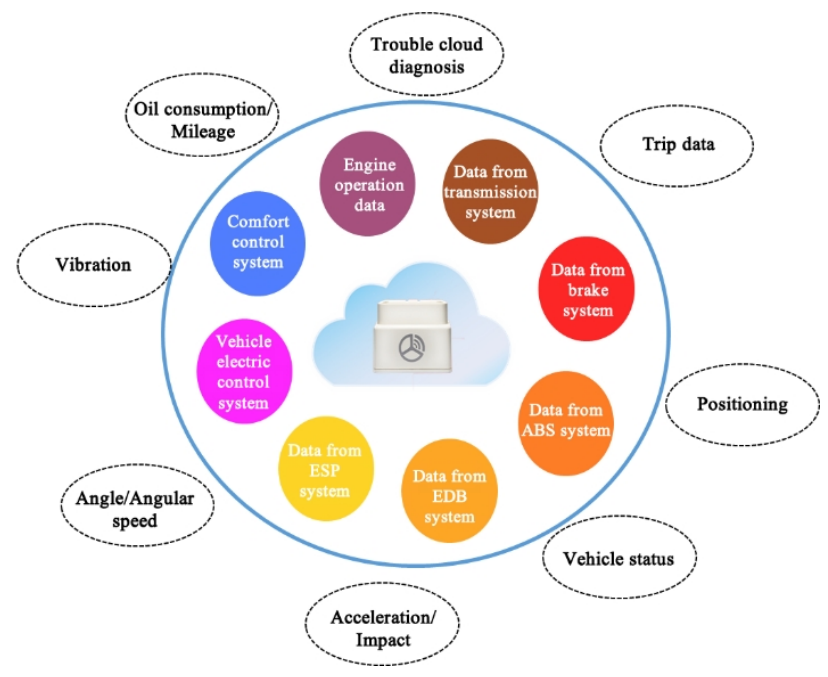

Fig.1 Vehicle OBD-Box function

We selected certain proportion of private cars to install the Intelligent Driving Box, which would upload the energy consumption and position data of cars at an interval of 1 10s. The Intelligent Driving Box consists of three functional modules, including the CAN bus data reading module, the satellite positioning module and the wireless communication module. It protects user 
privacies through the anonymous processing protocol. Intelligent driving data mainly include three main types: driving data, vehicle condition data and trips data.

\section{1) Driving data.}

They are classified into driving behavior data and real-time energy consumption data. The former include the count of sharp acceleration, count of sharp deceleration, over-speed and other data, and analyze the time and position of bad driving behaviors of drivers. The latter compass the collection time and instantaneous fuel consumption, collect the energy consumption data of cars in a real-time manner and analyze their emission contribution.

\section{2) Vehicle condition data.}

They mainly include diagnostic trouble codes (DTC) of the electronic control unit (ECU) and descriptions, battery voltage, fuel correction value, engine load and other basic data of the vehicle. The module monitors security hazards of different important parts of the car, identifies abnormalities of the car and ensures the driving security of the driver.

\section{3) trips data.}

They are divided into range data and position data. The former include the ignition time data, flameout time data, driving range and vehicle speed distribution, and demonstrate the mobility, travel time, travel distance, commutation and other aspects of the car. The latter include the positioning time, latitude, longitude, direction and other data, and analyze daily mobility habits, commutation, workplace position, residential position and other aspects of the driver.

\section{Data processing flow}

Car trips data processing mainly includes five steps: data acquisition, underlying data processing, analysis of mobility characteristics, profound mining of mobility rule and application of analysis results (see Figure 2).

\section{1) Data acquisition.}

Acquire intelligent driving data after desensitization processing, and perform parsing and cleansing.

\section{2) Underlying data processing.}

Draw a trip that starts with the ignition time of the car and ends with the flameout time of the car. Every trip records includes times and positions of ignition and flameout, distance and unique car code as well as the mobility track within the entire trip, namely latitude, longitude and other fields every second.

\section{3) Analysis of mobility characteristics.}

Find the temporal sequence of every trip of the car based on the unique code and determine the daily trips track of the car. Match OD points in the trip information with points of interest on the map, then match points of the trips track with the road network, and obtain quantitative indicators measuring trips characteristics of the car after data analysis.

\section{4) Mine trips rule in depth.}

Match the trip information with the road network to acquire daily mobility data of the car. Define the algorithm identifying travel destinations, screen trips based on destinations, and then forecast groups of users based on the frequency or rule of appearance of destinations. Calculate the nonlinear factor of the line based on the actual distance of daily travel and the linear distance of the OD point. 


\section{5) Application of analysis result.}

The Intelligent Driving Box can acquire mobility data of cars quickly and accurately, compared to the traditional traffic data survey. With intelligent driving data, we can parse trips characteristics of cars and users in depth and accurately, and support the effective implementation of traffic organization and traffic policies.

\section{Analysis of trips Characteristics of Cars}

The fifth comprehensive urban traffic survey of Beijing in 2014 (hereafter referred to as the "general survey") demonstrates[11] that the trips with car constitutes $32.1 \%$ of the means of mobility, excluding walking, within the Sixth Ring Road, while this figure is $31.5 \%$ in the central urban district, indicating the trips with car takes a proportion of nearly one third in all the means of mobility. Therefore, accurate analysis of tris characteristics of cars and identification of their inherent rule of trips will be of great importance to mitigate the traffic congestion.

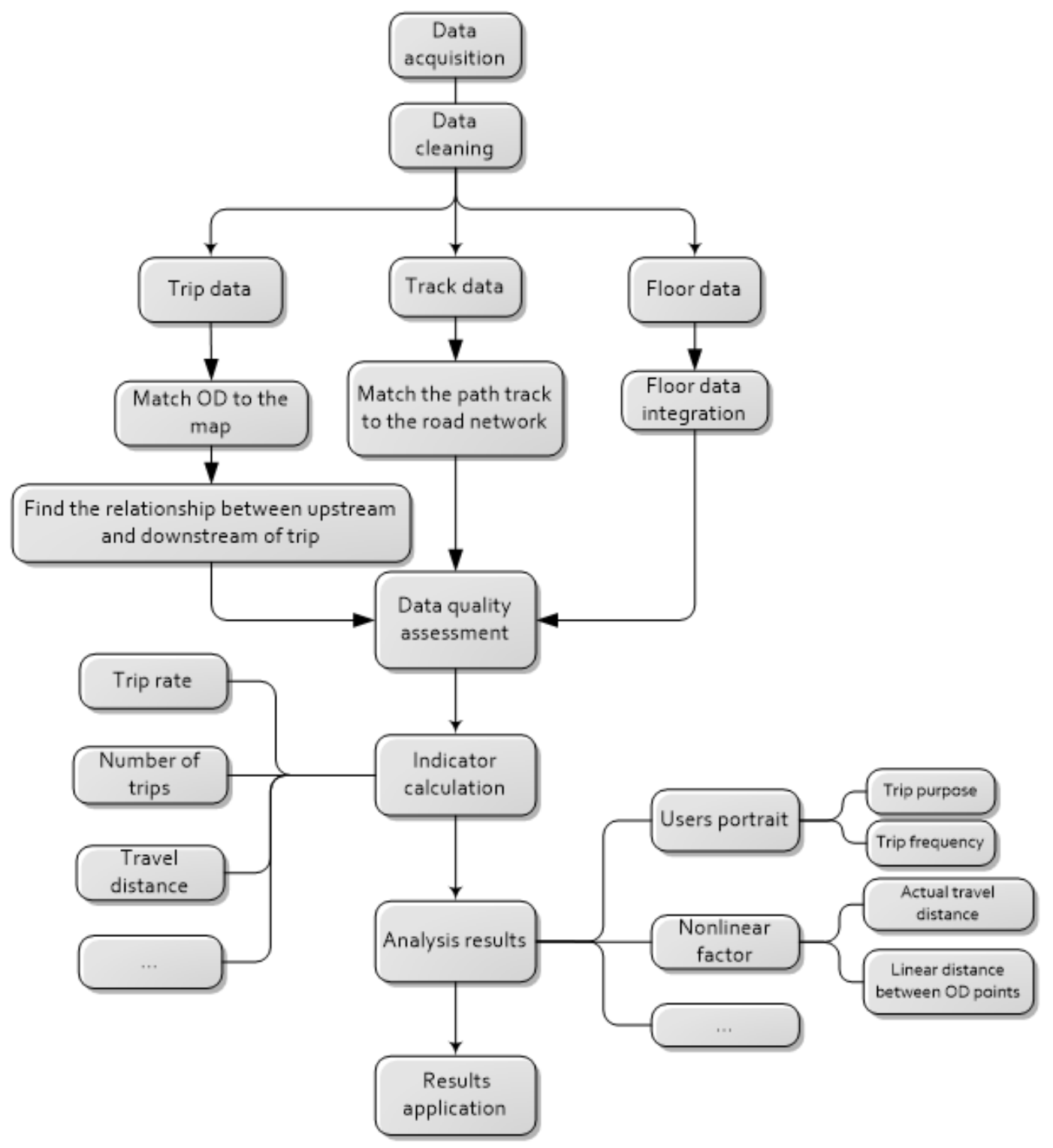

Fig. 2 Travel characteristics analysis process 


\section{Changes of car use rate}

\section{1) Concept of car usage rate.}

The car usage rate means the ratio of cars with trips to the total number of cars. We can employ intelligent driving data to calculate the car usage rate, which can reflect the operation level of the road network and the usage intensity of private cars.

\section{2) Relation between car usage rate and traffic congestion.}

The traffic performance index can visibly reflect and measure the road traffic congestion. Now, it has been widely used in many Chinese cities. We can compare the monthly average car usage rate and traffic performance index of Beijing in 2016 (see Figure 3). In February, the car usage rate and the traffic performance index both came at a low level as a result of the Spring Festival holiday. During the summer vacation in July-August, both indicators remained low. In September, a new term of all schools started, and both indicators hit the highest level, affected by the Mid-Autumn Festival and the National Day Festival that came together. We can see that the car usage rate and the traffic performance index display the similar trend, indicating usage behaviors of cars constitute a strong correlation with traffic congestion.

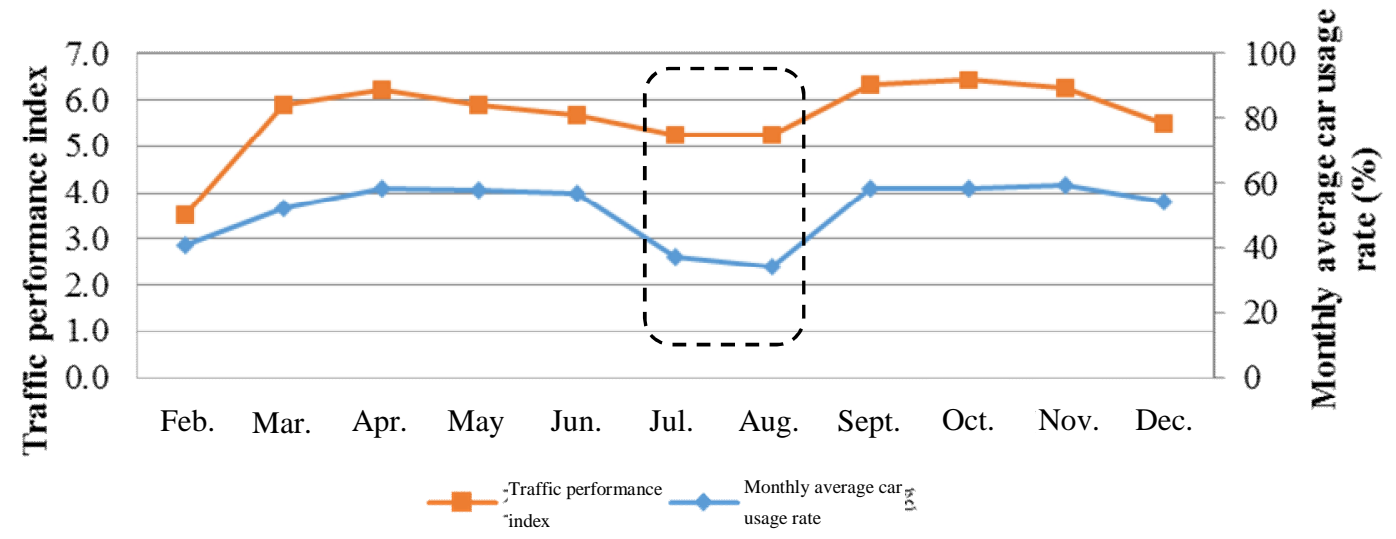

Fig.3 The Comparison between monthly average usage rate and monthly average peak traffic

performance index in 2016

\section{3) Impact of traffic policies on car usage rate.}

Beijing carried out the policy of traffic control based on two final numbers of vehicle registration numbers during working days in October 2008 and started the traffic control based on odd number and even number of vehicle registration numbers on special weathers since 2015 to mitigate the traffic and environmental pressure. We analyzed the daily average car usage rate in December 2016 (see Figure 4) and found that the average car usage rate was 60\% (which can be converted into $75 \%$ after deduction of the car use by one day every week) during the working days (the car use was reduced by one day every week), and that during the traffic control based on odd number or even number of vehicle registration numbers, the car usage rate of the entire road network fell down to $40 \%$. This demonstrates the traffic control made an obvious effect but the car usage rate was a bit higher than half of the mobility rate, namely $37.5 \%$, of $75 \%$, indicating the use of cars not under traffic control was increased to some extent after the traffic control based on odd number or even number of vehicle registration numbers. 


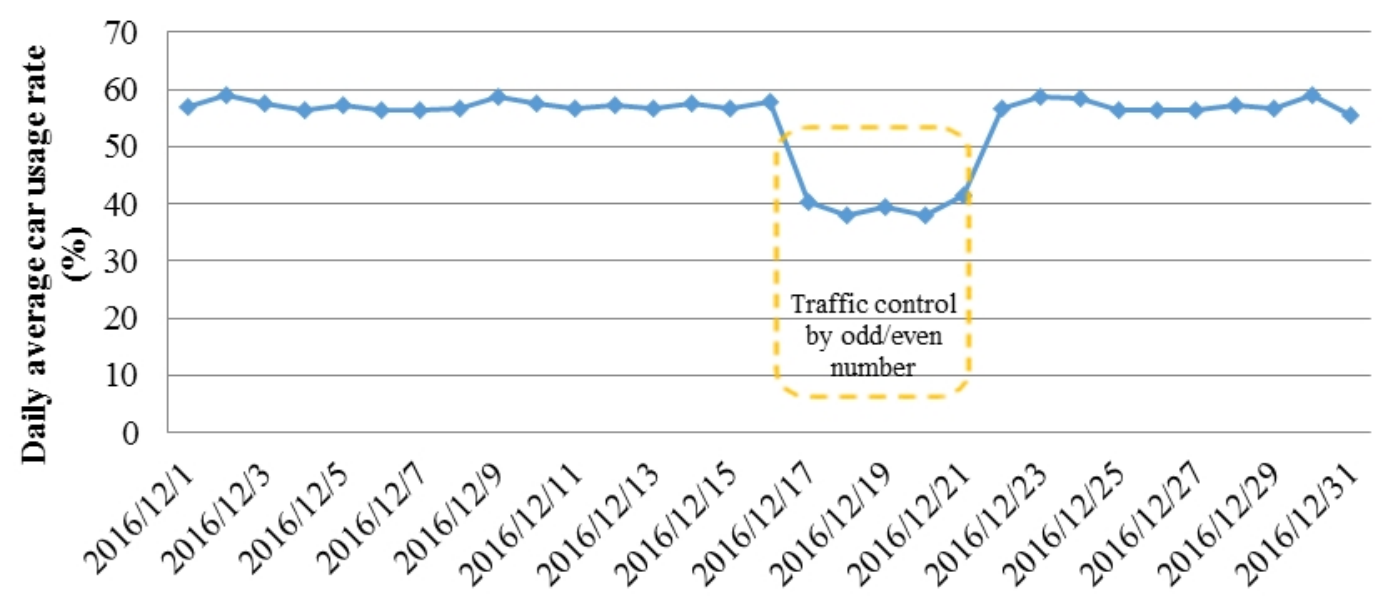

Fig.4 The influence of odd and even numbers restriction on trip rate in Dec. 2016

\section{4) Regional characteristics of car usage rate}

We analyzed the car usage rate (see Figure 5) based on administrative zones and found that the rate was relatively higher in the remote suburbs on the whole. Xicheng District recorded the lowest car usage rate at $51.3 \%$ and the biggest car base per 1,000 persons, up to 232 cars/1,000 persons-1, followed by Dongcheng District with corresponding figures of 50.9\% and 231 cars/1,000 persons- 1 . The two districts have a low car usage rate due to a relatively developed public traffic network, parking problem and other factors. However, the high car base per 1,000 persons and density of population lead to a high level of car mobility, which results in the congestion of the road network in the central urban districts.

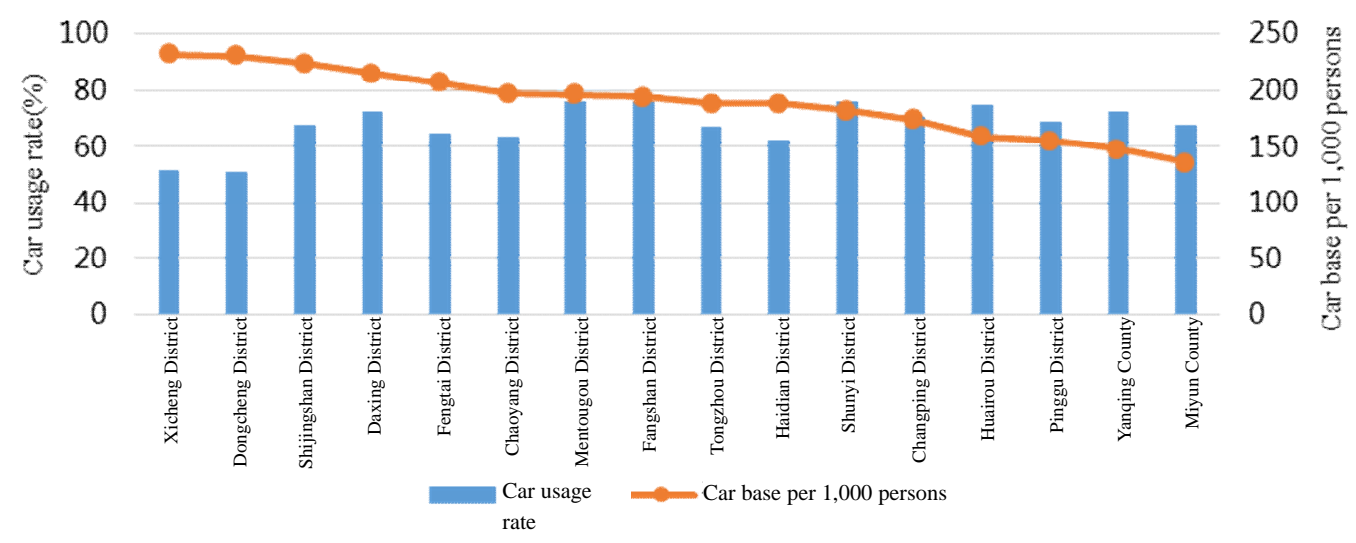

Fig.5 Car usage rate and the number of cars per thousand people in different areas

We also analyzed the car usage rate based on ring road (see Figure 6). The usage rate of private cars for all purposes declines from $71.5 \%$ outside the Fifth Ring Road to $52.0 \%$ within the Second Ring Road, demonstrating that the outer areas of the city depend on cars to a big extent, and the urban districts depend on cars to a small extent, due to the developed public traffic. 


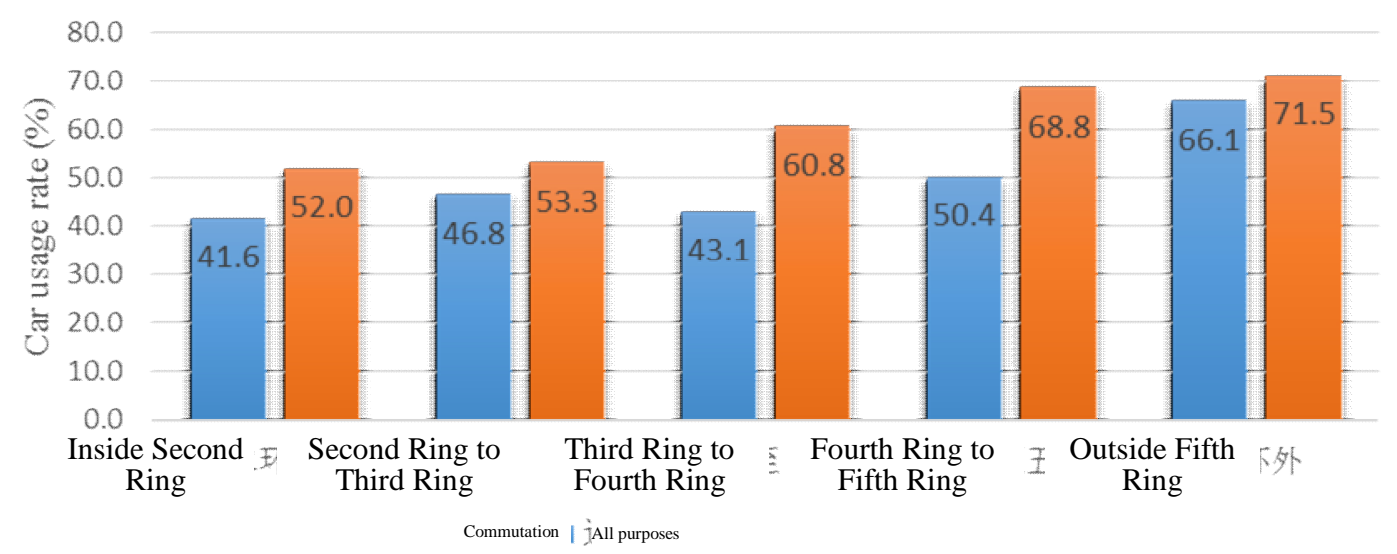

Fig.6 Car usage rate in different loop areas

We compared the car usage rate output from the intelligent driving data and the result of the general survey. The car mobility rate was $62 \%$ within the Sixth Ring Road during the working days in 2014, and the daily average car mobility rate was 60\% in December 2016 according to the intelligent driving data. The two figures are basically the same and prove that the analysis of car trips characteristics based on intelligent driving data is representative to some extent.

\section{Average trips of cars per day}

The average trips of cars is one of the indicators measuring the usage intensity of cars and also an important parameter assessing the extent of controllability of car use. We analyzed the daily average trips of sample cars of Beijing in February 2016, the Spring Festival holiday (see Figure 7): The daily average trips of cars kept relatively stable at 4.8/day-1 during non-holiday days, kept rising to 5.3/day-1 during the week before the Spring Festival (February 1-6) and arrived at the peak value of 5.76/day-1 one day before the Spring Festival (the twenty-eighth day of the lunar December). The indicator edged down with an average value of $4.7 /$ day-1 during the Spring Festival holiday and arrived at the valley of 4.42/day-1 on February 8 (the first day of the lunar January).

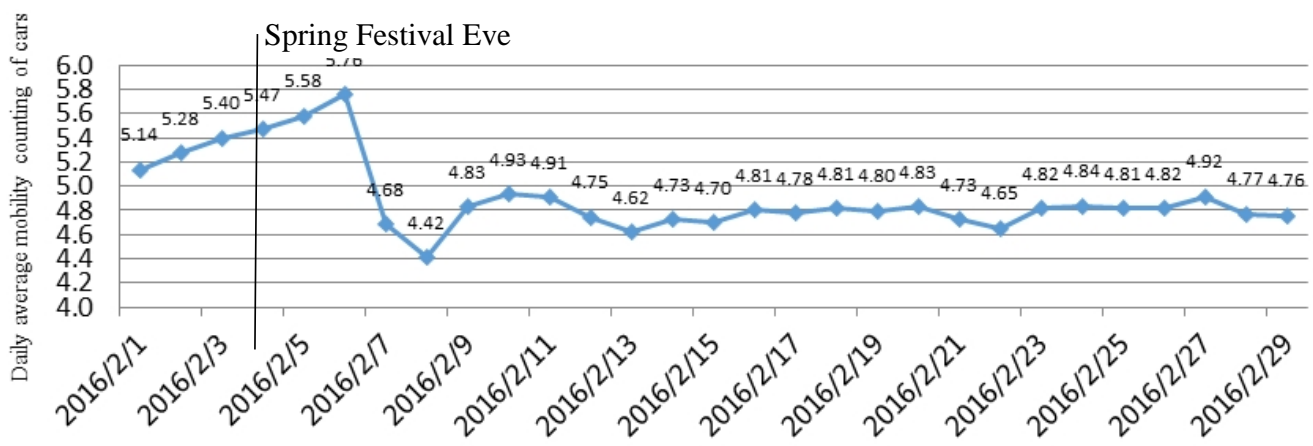

Fig.7 The number of daily trips during the Spring Festival in Feb.2016

\section{Mobility distance of cars}

An increase in the trip distance of cars will bring an increase in the turnover and the load of the road network. The trip distance varies against different holidays and traffic control policies. 
In Beijing, the average trip distance is $11.4 \mathrm{~km}$, and we can see that the trip distance obviously rose during the holiday and the average mobility distance was $12.4 \mathrm{~km}$ during the National Day holiday after comparing it to the trip distance during the holiday (see Figure 8). The daily average trip distance presents a cyclical gradual growth from Monday to Friday.

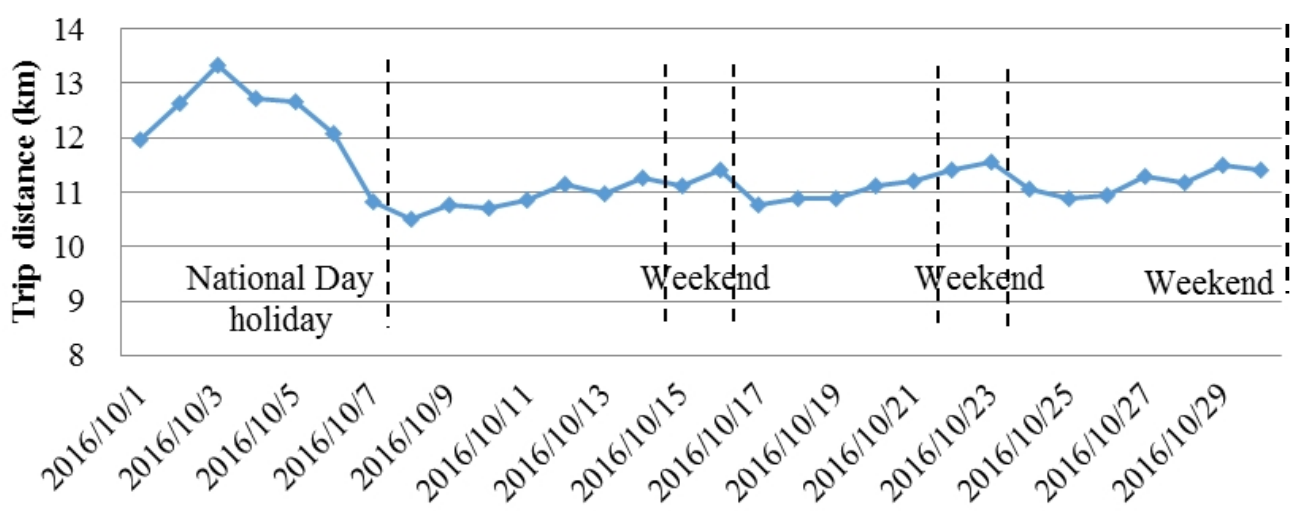

Fig.8 Car daily average trip distance in Oct.2016

䈀e can see from Figure 9 that during the traffic control based on odd number or even number of vehicle registration numbers, the daily average mobility distance obviously rose and came at an average value of $12.6 \mathrm{~km}$, an increase of $1.2 \mathrm{~km}$ compared to normal days. Further, we can infer from Figure 4 that during the traffic control based on odd number or even number, the daily average car trip rate obviously declined, but the daily average trip distance evidently rose.

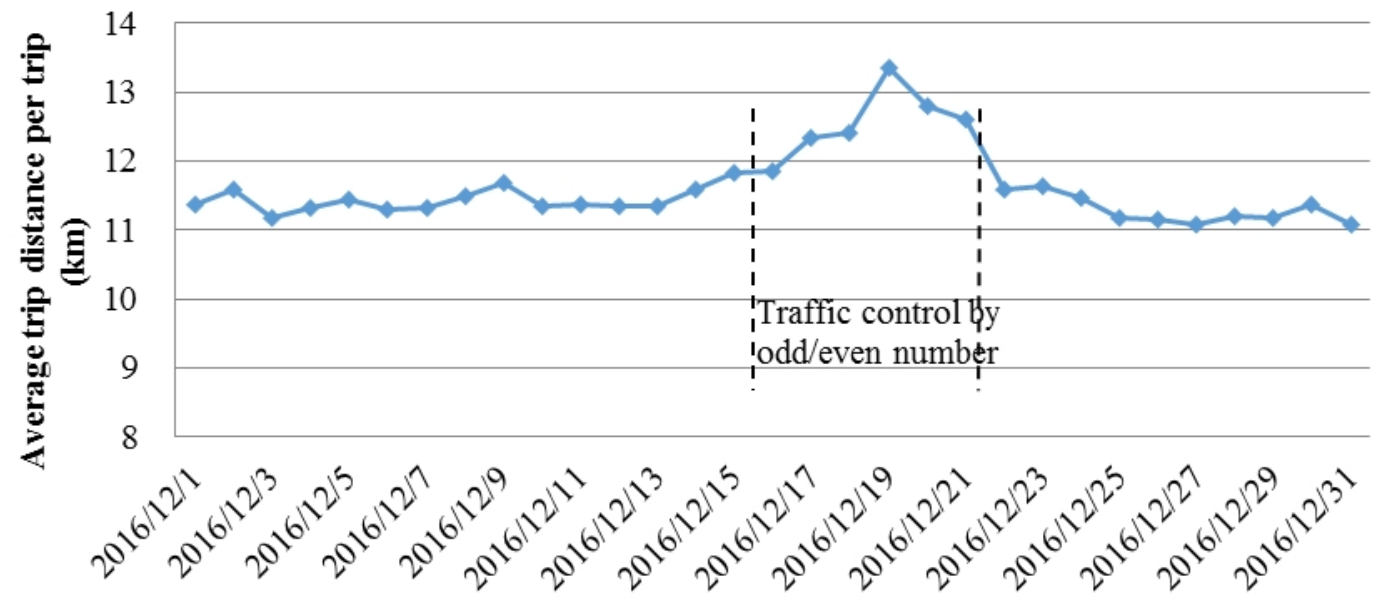

Fig.9 Car daily average trip distance in Dec.2016

Figure 10 illustrates the average trip distances between different ring roads. The overall trip distance for commutation was higher than the trip distance for all purposes. Moreover, the farther the area is away from the downtown, the longer the trip distance will be. The trip distance for commutation was $13.7 \mathrm{~km}$ outside the Fifth Ring Road, which posed a huge pressure on the road network in the central urban districts. 


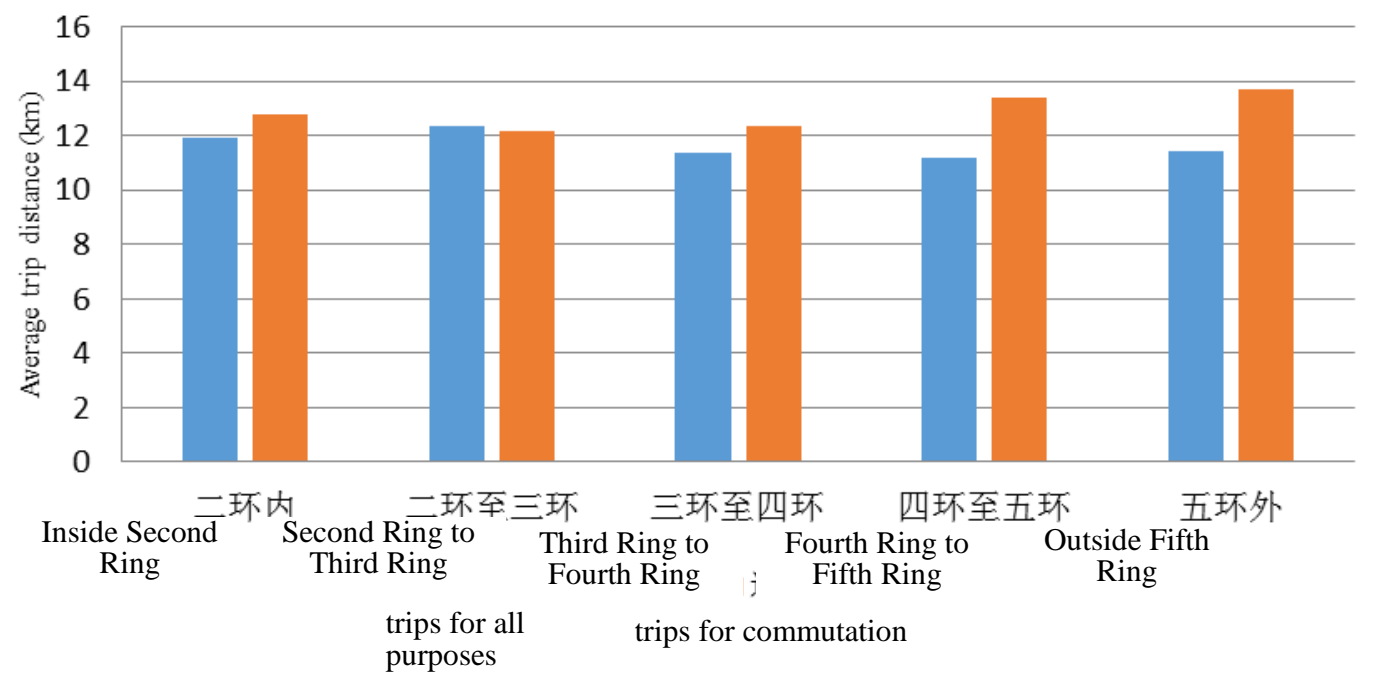

Fig10. Trip distance in different ring road areas

\section{Mine Trip patterns in Depth}

Besides directly calculating the indicators measuring car trip patterns, we can also mine intelligent driving data in depth to assess characteristics of drivers, usage of the road network and other traffic indicators.

\section{Portrait of car users}

We can judge users are office workers, overtime workers, parents picking up children or special car drivers or fall into other types, according to average trips per day, trip distance, travel time, travel purpose and other information of car users.

Commuting workers have regular places of residence and work and travel regularly every day. As illustrated in Figure 11, the driver frequently travels back and fro between Guanganmennei Street and Jiuxianqiao, and the trip between his residence and workplace accounts for $60 \%$ of his total trip distance. This demonstrates obvious commutation characteristics, and we can judge the driver is an commuting worker.

We screened the parameters for trip characteristics of all commuting workers (see Figure 12) and found that the overall trend of the starting time of office workers is consistent with that of all types, but commuting workers travel in a more concentrated manner during the traffic peaks in the morning and in the afternoon and start out 30 minutes earlier than the traffic peak in the morning. 


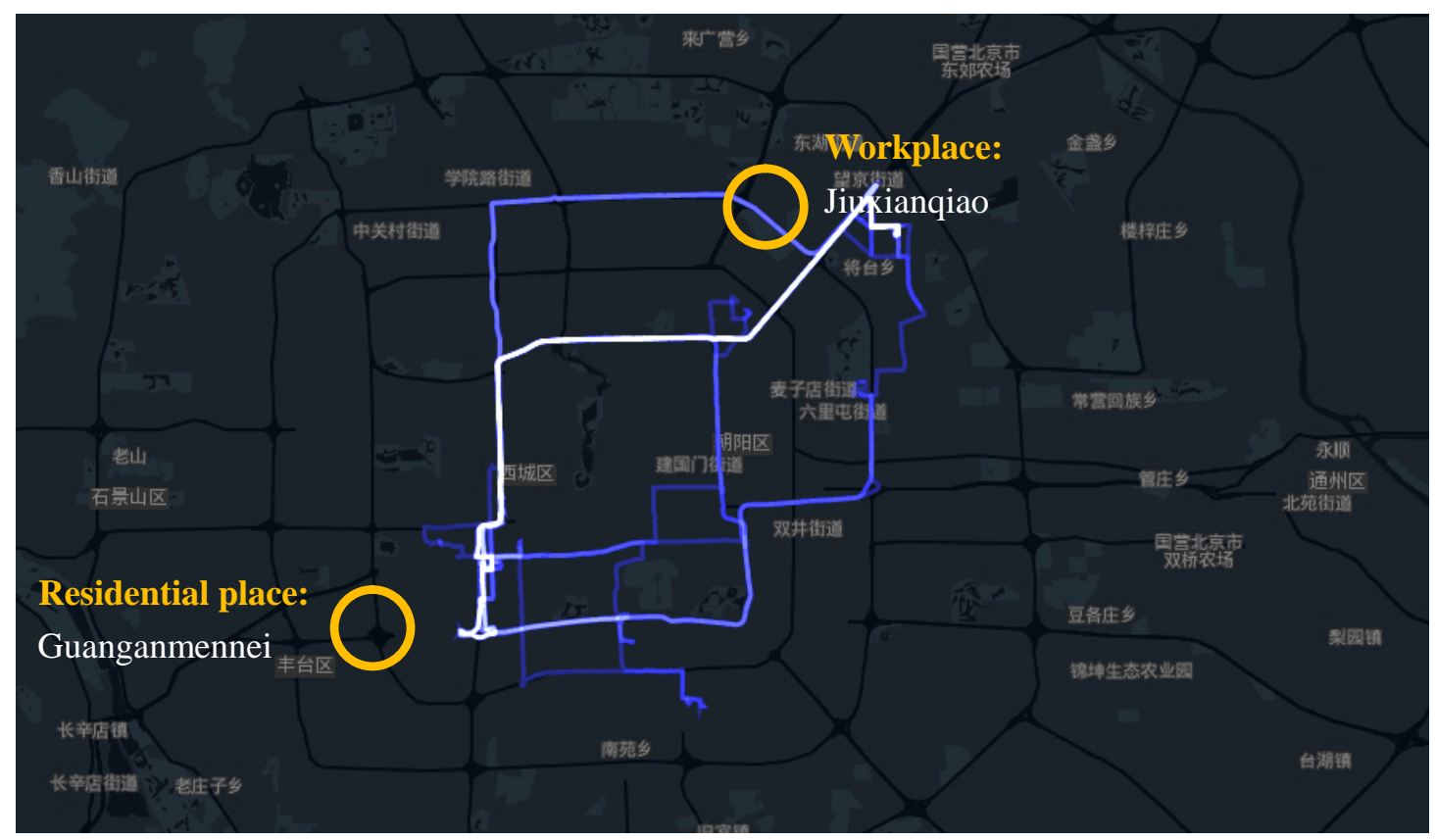

Fig.11 Commuting route for an office worker

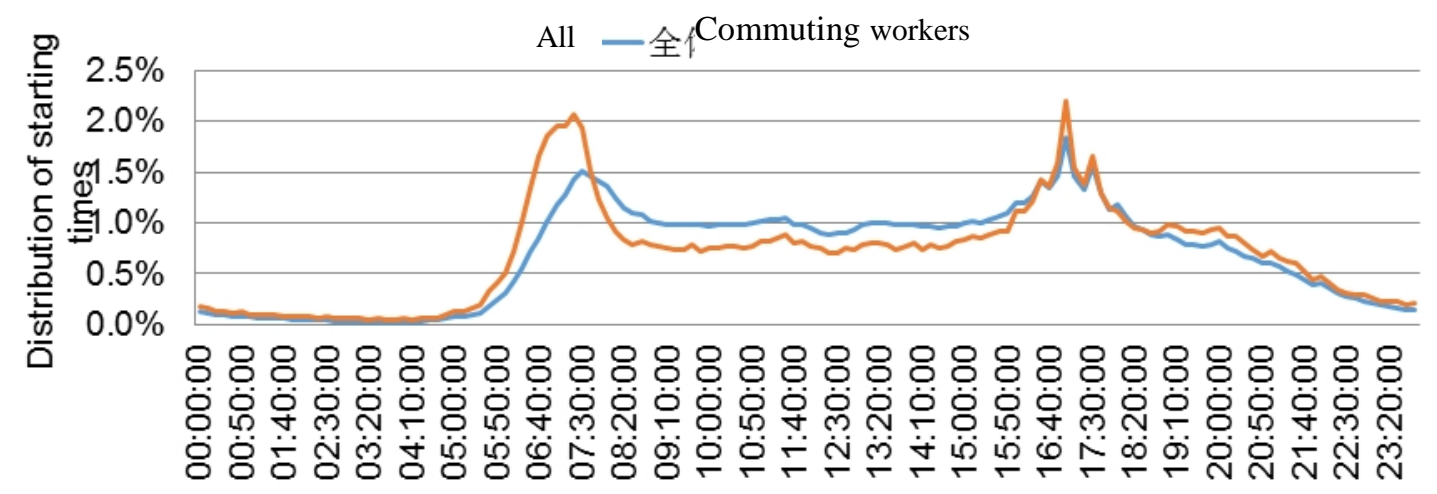

Fig.12 The proportion of departure time for Commuting workers

\section{Nonlinear factor}

The nonlinear factor is the ratio of the actual driving distance and the spatial linear distance between two OD points of the mobility line. The nonlinear factor should not be higher than 1.4[12] for public traffic lines. However, the detour distance is greatly increased as a result of the lack of axial express roads and ring connecting lines as well as the unsound road network in some areas. At present, the factor is 1.68 for the entire road network in Beijing, indicating a long detour distance.

We compared the nonlinear factors of different administrative zones (see Figure 13). The mobility within an administrative zone refers to the mobility with all the tracks falling into the administrative zone. Overall, the nonlinear factors are relatively high in the six urban districts and Mentougou District. The factor is the highest, up to 1.70, for Chaoyang District, 1.68 for Dongcheng District, Haidian District, Shijingshan District and Mentougou District, and the lowest at 1.45 for Yanqing County. 


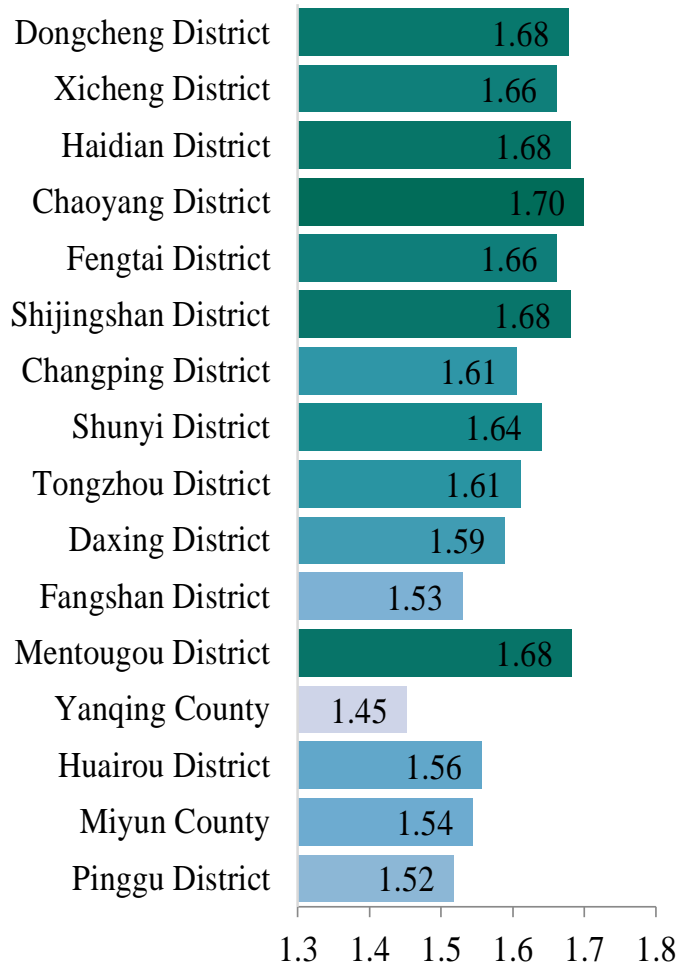

Fig.13 The non-linear coefficient of travel in different administrative areas of Beijing

\section{Conclusion}

OBD data can well reflect behavioral characteristics of cars in the entire mobility process and first realize dynamic track of mobility behaviors of cars. In this paper, we have analyzed the intelligent driving data of Beijing in 2016, demonstrated the method to analyze overall characteristic indicators for car trips based on OBD data, and performed quantitative assessment of the implementation effect of the traffic policy. In addition, we can mine big data of car trip patterns in depth to collect mobility information in greater depth, including the information required to perform precise portrait of users, identify user types, describe mobility tracks, assess the structure of the road network and perform real-time monitoring over the parking demand of the road network. Moreover, we can also employ OBD data to enable real-time monitoring over vehicle emissions and analyze behavioral characteristics of drivers. In future, we will integrate these data into other traffic big data for analysis, mine more trip characteristics, assess their impact on traffic operation, analyze the congestion reason in depth, and support the lean mobility management of cars and policymaking.

\section{Acknowledgements}

Receiving Date: August 15, 2017

Fund Project: The "Traffic Data Mining Team" (Z161100004916021) Project under the Beijing 100 Scientific \& Technological Pioneer Cultivation Plan, the "Key Technology and System for Beijing-Tianjin-Hebei Traffic Analysis Based on Pseudo Code Signaling Data" (Z161100005116006) under the Beijing Scientific \& Technological Plan and the "Pilot Platform for Integration and Unified Publishing of Beijing Road Condition Information” (Z161100001116094) under the Beijing Scientific \& Technological Plan 
About the Author: Sun jianping(1978- ),female, a native of QinHuangdao, Hebei, Doctor, professorial senior engineer, Deputy Director of ITS department, Beijing Transport Institute. Major research domains: Traffic planning and intelligent traffic. E-mail: sunjp@ bjtrc.org.cn

\section{References:}

[1]Zhang Tianran. Job-Housing Spatial Distribution Analysis in Shanghai Metropolitan Area Based on Cellular Signaling Data[J]. Urban Transport of China, 2016, 14(1): 15-23.

[2] Ma Chunjing. Study on Methods of Analyzing the Floating Population Trip Characteristics Based on Phone Signaling Data[D]. Nanjing: Southeast University, 2016.

[3] Xu Wenyuan, Deng Chunyao, Liu Baoyi. Approach on Public Traffic Passenger Flow Statistics Based on IC Data[J]. China Journal of Highway \& Transport, 2013, 26(5): 158-163.

[4] Zuo Kuaile. Research on Passenger Flow Forecasting Method and Application in Different Level of Time Based on IC Smart Card Data[D]. Nanjing: Southeast University, 2016.

[5] Wu Peili, Liu Kuien, Hao Shengang, et al. Rapid Traffic Congestion Monitoring Based on Floating Car Data[J]. Journal of Computer Research and Development, 2014, 51(1): 189-198.

[6] Wan Xia, Chen Jun, Wang Wei. Analysis the Car Trip Characteristics of Clustered City[J]. Urban Planning Forum, 2007(3): 86-89.

[7] Gao Feng, Guo Yanyun, Chen Jinchuan. Study on the Travel Attributes of Private Cars on the Basis of Home-Based Trip Chains[J]. Journal of Transportation Systems Engineering \& Information Technology, 2011, 11(s1): 132-139.

[8] Liu Mingjun, Guo Jifu, Gao Liping, et al. Analysis and Modeling of Private Car Usage through Travel Behavior[J]. Journal of Jilin University(Engineering and Technology Edition), 2009, 39(s2): $25-30$.

[9] Wu Wen, Shuai Bin, Yin Huanhuan, et al. Study of Travel Behavior by Car Based on BL Model[J]. Journal of Transportation Engineering \& Information, 2012(3): 71-75.

[10] Wen Ping, Zhao Pengjun. Car Use in the Historic City Centre of Beijing: An Empirical Analysis and Integrated Planning Proposal[J]. Urban Planning International, 2014, 29(2): 52-58.

[11] Beijing Transport Institute. General Report on Fifth Comprehensive Survey of Urban Traffic in Beijing [R]. Beijing: Beijing Transport Institute, 2016.

[12] GB 50220-95 Urban Road Traffic Planning and Design Specification [S]. 